\title{
Radiolésions aiguës localisées
}

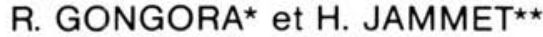

\begin{abstract}
RÉSUMÉ
Les radiolésions aiguës localisées sont relativement rares. Elles ont, en général, une origine accidentelle. Elles constituent une entité anatomoclinique. L'évolution clinique et la conduite thérapeutique dépendent du niveau et de la distribution topographique des doses. L'évaluation dosimétrique fait appel à des méthodes physiques et à des examens paracliniques. L'étude de 60 cas suivis au Centre international de radiopathologie (Institut Curie - Institut de protection et de sûreté nucléaire du CEA) permet de décrire la symptomatologie clinique et d'évoquer les problèmes posés au médecin radiopathologiste par les radiolésions aiguës localisées.
\end{abstract}

\section{ABSTRACT}

Local acute radiation injuries do not occur very often. Their origin is generally accidental. They show specific anatomo-clinical features. The clinical evolution and therapeutic behaviour are dependent on the dose level and topographical distribution. The dosimetric assessment requires physical methods and paraclinical investigations. From a study of 60 cases followed by the International center of radiopathology, the clinical symptomatology is described and the problems raised to the radiopathologist physician by local acute radiation injuries are stated.

\section{GÉNÉRALITÉS}

Les radiolésions aiguës localisées constituent une entité anatomoclinique. Elles se distinguent des brûlures thermiques et électriques et des autres lésions dues aux rayonnements ionisants. Les manifestations cliniques, d'une part, peuvent être retardées par rapport au moment de P'exposition, d'autre part, ne sont pas simultanées, mais apparaissent successivement et donnent lieu à une évolution en plusieurs temps. Ceci est dû au fait que pour un certain niveau de dose, les rayonnements entraînent, non pas la mort cellulaire intermitotique, mais l'arrêt de la multiplication cellulaire (mort mitotique).

Les conséquences sont différées et le délai d'apparition des lésions est, en majeure partie, conditionné par les durées de vie respectives des différents types cellulaires. Ainsi, les conséquences de l'arrêt de la division cellulaire au niveau de la couche basale de la peau, se manisfestent

* Centre international de radiopathologie, Organisation mondiale de la santé, Service de radiopathologie, Institut Curie, 26, rue d'UIm, 75005 Paris.

** Commissariat à l'énergie atomique, IPSN, Département de protection, BP 6. 92260 Fontenay-aux-Roses. 
trois semaines après l'exposition, tandis que les conséquences de l'irradiation de l'endothélium vasculaire sont beaucoup plus tardives, de l'ordre de plusieurs mois.

Les radiolésions aiguës localisées se traduisent donc souvent par: une phase de latence, une phase d'évolution aiguë, une phase d'évolution chronique.

La dose nécessaire pour produire une radiolésion aiguë localisée est très supérieure à la dose létale pour une irradiation globale. Schématiquement, la dose entraînant une ulcération est supérieure à 1800 rads (18 Gy) pour une exposition unique de courte durée, alors que la dose létale $50 \%$ pour une irradiation globale aiguë est de l'ordre de 350 rads (3,5 Gy).

L'évolution, le pronostic, le traitement dépendent essentiellement de la dose délivrée aux tissus. Aussi est-il absolument nécessaire de déterminer les doses. A cet égard, la dosimétrie physique est indispensable ; mais elle n'est pas toujours en mesure de fournir des valeurs absolues de doses et il est nécessaire d'avoir recours à des méthodes biologiques d'évaluation dosimétrique. Le traitement, essentiellement chirurgical, est guidé par les données dosimétriques.

\section{2. ÉTIOLOGIE ET FRĖQUENCE}

Plus de 200 malades ayant subi une irradiation externe ont été suivis dans le Service de radiopathologie de l'Institut Curie, en étroite collaboration avec le Département de protection du CEA.

La nature des rayonnements et les circonstances d'exposition étaient les suivantes:

\begin{tabular}{|c|c|}
\hline$x$ & Radiodiagnostic général \\
\hline $\mathrm{x}$ & Radiodiagnostic dentaire \\
\hline $\mathrm{x}$ & Radiothérapie \\
\hline $\mathrm{x}$ & Cristallographie et travaux expérimentaux \\
\hline$\gamma^{192} \mathrm{Ir}$ & Gammagraphie industrielle \\
\hline$\gamma^{137} \mathrm{Cs}$ & Gammagraphie industrielle \\
\hline$\gamma{ }^{226} \mathrm{Ra}$ & Curiethérapie \\
\hline$\gamma{ }^{60} \mathrm{Co}$ & Radiothérapie \\
\hline “ ” & Usage industriel expérimental \\
\hline “ " & Chargement d'irradiateur médical \\
\hline “ ” & Chargement d'irradiateur industriel \\
\hline “ " & Irradiation industrielle \\
\hline neutrons $+\gamma$ & Réacteurs expérimentaux \\
\hline électrons & Accélérateurs expérimentaux \\
\hline neutrons & Accélérateur expérimental \\
\hline protons & Accélérateur expérimental \\
\hline
\end{tabular}


Il est à noter labsence d'accident ayant entraîné une irradiation sérieuse dans les centrales nucléaires en exploitation industrielle.

Parmi ces 209 sujets, 60 ont présenté des radiolésions aiguës localisées sévères ayant nécessité une thérapeutique majeure.

L'analyse porte sur ces 60 malades dont 13 présentaient, en outre, un syndrome d'irradiation globale qui, dans 3 cas, a dominé le tableau clinique (à titre indicatif, durant la même période, 10 malades ont été traités pour irradiation globale aiguë sans surexposition locale).

Pour ces 60 malades, les sources de rayonnement étaient les suivantes :

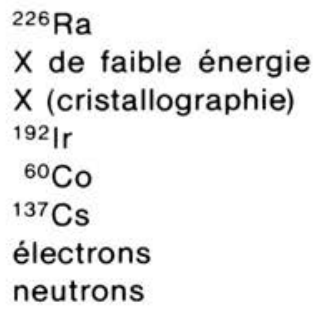

\section{DOSIMÉTRIE PHYSIQUE}

La gravité des lésions dépend de la dose et de sa répartition topographique. La détermination physique de la dose est indispensable; elle est pratiquée de manière systématique. La dosimétrie physique repose sur trois paramètres fondamentaux: les caractéristiques physiques de la source, la géométrie source-sujet, la durée d'exposition.

Les caractéristiques physiques sont généralement bien connues. II s'agit le plus souvent de photons $X$ ou $\gamma$, quelquefois d'électrons, exceptionnellement de neutrons. En ce qui concerne les photons, pour les $X$, il s'agit : soit de rayons X émis sous des tensions de 20 à $50 \mathrm{kV}$, soit de rayons $X$ de très faible énergie ( 4 à $12 \mathrm{keV}$ ); pour les photons $\gamma$ il s'agit : de ${ }^{60} \mathrm{Co}(1,17-1,33 \mathrm{MeV})$, de ${ }^{192} \operatorname{Ir}(0,3 \mathrm{MeV})$, de ${ }^{137} \mathrm{Cs}(0,662 \mathrm{MeV})$, de ${ }^{226} \mathrm{Ra}$.

En ce qui concerne les électrons, il s'agit, dans deux cas, d'électrons de $4 \mathrm{MeV}$ et, dans un cas, d'électrons de faible énergie. Dans les cas d'irradiation par neutrons et $\gamma$ à partir d'un réacteur expérimental, les caractéristiques étaient également parfaitement connues.

La géométrie source-sujet peut être constante ou variable. Dans les cas d'exposition longue, elle est souvent variable; dans les cas d'exposition de brève durée, elle est, en général, constante. La distance conditionne le rapport entre la dose locale et la dose globale moyenne; ce rapport est d'autant plus élevé que la distance est plus faible. Dans la plupart des cas, la distance est très faible, voire nulle, la préhension d'une source radioactive étant une circonstance fréquente. Par ailleurs, il est important, lors de l'évaluation dosimétrique, de prendre en considération 
les caractéristiques morphologiques individuelles. Diverses techniques sont utilisées dans ce but (mesures par conformateur, moulage des mains, tomodensitométrie).

Le troisième paramètre dosimétrique est la durée d'exposition. Cette durée peut être variable, d'une fraction de seconde à plusieurs heures. Lorsque la durée d'exposition est courte, son évaluation est difficile ; l'appréciation subjective peut varier d'un facteur 10. En revanche, la géométrie source-sujet est le plus souvent constante. A l'inverse, lorsque la durée d'exposition est longue, l'erreur sur le temps est faible mais la géométrie est souvent variable.

Ces paramètres sont pris en considération lors de la reconstitution des conditions d'exposition. Des mesures sont effectuées à l'aide de dosimètres appropriés. Les résultats acquis directement ou après traitement par calculateur permettent l'établissement de cartes dosimétriques, en général sous la forme de courbes isodoses.

Lorsque tous les paramètres sont connus, on obtient des résultats dosimétriques absolus. Mais c'est rarement le cas; en effet, la durée est souvent difficile à évaluer et l'on ne dispose alors que d'une dosimétrie relative. Enfin, lorsque la géométrie a varié pendant l'exposition de manière incontrôlée, on est amené à proposer les résultats obtenus par les mesures et par le calcul pour les hypothèses les plus probables. Différents modèles peuvent être proposés, qu'il convient alors de confronter à des données cliniques et biologiques.

C'est pourquoi, parallèlement à la dosimétrie physique, on s'efforce de recueillir le maximum de renseignements cliniques et biologiques qui dépendent de la dose. Malheureusement, nous ne disposons pas actuellement de dosimètre biologique parfait, mais on utilise un certain nombre d'indicateurs.

\section{INDICATEURS CLINIQUES ET PARACLINIQUES}

\subsection{Généralités}

La précocité de signes cliniques tels que l'érythème, l'œdème, les phlyctènes, signifie que l'irradiation est très importante. Mais souvent, ces manifestations cliniques sont latentes et, seul, le recours à des investigations paracliniques permet de prévoir la gravité de la situation. Ces examens ont pour but de déceler les modifications infracliniques au niveau de la peau, du tissu conjonctif, des vaisseaux et du squelette. Ils sont aussi utiles pour la surveillance de l'évolution.

Les méthodes les plus intéressantes sont :

- les méthodes thermiques: thermométrie, thermographie par caméra ou en plaque par cristaux liquides, effusivimétrie ;

- les méthodes radioisotopiques: scintigraphie vasculaire, clairance sous-cutanée, scintigraphie osseuse ;

- les méthodes physicochimiques: mesure du chlore cutané, mesure du $\mathrm{pH}$ cutané, fluxmétrie, évaporimétrie, capillaroscopie, effet Doppler. 
Trois de ces méthodes, en particulier, fournissent des renseignements de grande valeur: la thermographie, la scintigraphie vasculaire, l'effusivimétrie. Les deux premières présentent, en outre, l'avantage de fournir des cartes isothermes ou d'isoactivité qu'il est très commode de comparer, d'une part aux courbes isodoses de la dosimétrie physique, d'autre part aux lésions à la période d'état.

\subsection{Thermographie}

Les résultats de la thermographie présentent un intérêt majeur. En effet, dès l'exposition, pendant la période de latence clinique et parfois longtemps avant l'observation des premiers signes cliniques, ils mettent en évidence une importante hyperthermie des territoires exposés (fig. 1). Les différences de température entre régions symétriques peuvent atteindre plusieurs degrés. Ce phénomène, indice de gravité, est important à saisir dès le premier jour, car il peut s'estomper par la suite. Souvent, à ce stade initial, les courbes isothermes circonscrivent pratiquement les volumes irradiés.

La thermographie semble moins utile pendant la phase d'évolution aiguë. Elle retrouve un regain d'intérêt pour la surveillance de l'évolution à court et moyen terme, les rechutes étant précédées et s'accompagnant d'une élévation thermique.

Enfin, la guérison s'accompagne d'une tendance à la normalisation des réponses thermographiques.

La thermographie présente, toutefois, des limites; on observe des fluctuations physiologiques importantes et il n'est guère possible, dans l'état actuel d'exploitation de nos données, de définir des valeurs absolues de référence. D'autre part, on se réfère généralement au côté symétrique, ce qui n'est pas possible dans le cas d'irradiation bilatérale des mains par exemple. II n'a pas été trouvé de corrélation entre la dose et les réponses thermographiques. On peut, en outre, observer une élévation thermique périlésionnelle et même à distance, vraisemblablement d'origine vasculaire réflexe, mais elle est faible.

\subsection{Scintigraphie}

La scintigraphie vasculaire consiste à enregistrer, à l'aide d'une caméra à scintillations reliée à un calculateur, le passage vasculaire, au niveau des régions examinées, d'un indicateur radioactif préalablement injecté par voie veineuse ou par voie artérielle. Des enregistrements séquentiels permettent de "visualiser" et de quantifier le passage vasculaire. Le traitement des données aboutit à l'obtention de courbes de passage vasculaire, au niveau de différents territoires définis a posteriori (fig. 2).

Les modifications de débit vasculaire sont précoces; elles précèdent les manifestations cliniques; elles peuvent être très importantes; le rapport du débit entre zone irradiée et zone saine symétrique peut être de 10. Mais ce rapport n'a qu'une valeur indicative car il n'est pas corrélé avec la dose. 


\subsection{Effusivité}

L'effusivité est la propriété que présente un milieu d'échanger de la chaleur avec un autre milieu avec lequel il est en contact. Cette propriété est caractérisée par un coefficient d'arrachement thermique ou effusivité. La méthode du Touchau permet la caractérisation thermique atraumatique des tissus. II est possible d'explorer les tissus sur une profondeur d'une dizaine de millimètres.

L'interprétation de courbes de zones pathologiques implique une comparaison avec des zones de référence en tissu sain. Dès le début de la phase de latence clinique, l'effusivimétrie montre des modifications discrètes de nature inflammatoire. Ces anomalies très précoces peuvent régresser au cours de cette phase de latence; il est donc important de procéder à la mesure dès le premier jour de l'exposition.

En l'absence de dosimétrie physique absolue, l'association de ces différentes techniques permet dans la majorité des cas de prévoir l'évolution.

\section{CLINIQUE}

\subsection{Généralités}

Les radiolésions aiguës localisées présentent des caractères cliniques spécifiques :

- Atteinte de tous les tissus (à des degrés divers, en fonction de la nature et de l'énergie des rayonnements).

- Seuil d'apparition des lésions; schématiquement, érythème entre 300 et 800 rads, épidermite sèche à 500 rads, épidermite exsudative entre 1200 et 2000 rads, nécrose à partir de 2500 rads.

- Temps de latence infraclinique,

- pour les doses entre 1200 à 2000 rads le temps de latence de l'épidermite exsudative est de 3 semaines,

- pour les doses qui induisent une nécrose, le temps de latence pour la nécrose peut être de 6 à 18 mois.

- Evolution souvent chronique.

- La gravité est liée à la dose et à sa répartition.

- Risque de cancer.

Les signes et l'évolution clinique offrent de multiples aspects. A travers la multiplicité et la complexité des manifestations cliniques se dégagent deux formes schématiques qui permettent de comprendre le "génie" de la maladie, une forme limitée à l'épithéliite exsudative, une forme avec endothéliite vasculaire.

La forme limitée à l'épithéliite exsudative comprend les phases suivantes : érythème précoce, phase de latence de trois semaines, érythème secondaire immédiatement suivi de l'épithéliite exsudative, et restauration qui s'effectue en 3 à 6 mois, soit avec séquelles trophiques, soit sans séquelles. 
Dans la forme avec endothéliite vasculaire, on observe, comme dans la précédente, l'érythème précoce, une phase de latence, un érythème secondaire, l'épithéliite exsudative et une restauration apparente. Mais après plusieurs mois de silence clinique, l'évolution reprend de manière explosive avec ulcération profonde et nécrose.

Nous envisagerons successivement la symptomatologie clinique, les formes cliniques.

\subsection{Symptomatologie}

Elle est très riche; on observe des signes physiques : érythèmes, œdèmes, phlyctènes, ulcérations, nécroses, scléroses, troubles thermiques. Sur le plan fonctionnel, on note une sensation de chaleur initiale, des paresthésies, des troubles de la sensibilité tactile et thermique, un syndrome douloureux.

\subsubsection{Signes physiques}

\section{L'érythème}

L'érythème est fréquent. II implique une dose minimale de 300 à 800 rads à la peau. La chronologie d'apparition présente un intérêt pronostique. II peut être précoce ou tardif. Précoce, il peut être transitoire, fugace mais riche de signification; il est le présage d'une évolution sévère ; il est donc très important de le repérer. II peut apparaître dès l'exposition ou quelques heures après l'exposition. L'érythème qui apparaît trois semaines après l'exposition précède immédiatement l'épithéliite exsudative. L'érythème peut se manifester plus tardivement, de 6 mois à 18 mois; il annonce puis accompagne les poussées de "vascularite" avec œdème et syndrome douloureux.

\section{L'œdème}

L'œdème est fréquent. II intéresse le plus souvent le territoire exposé mais s'étend parfois au-delà. La topographie des œdèmes précoces fournit une indication sur le volume irradié mais ne permet pas d'en définir les limites avec précision.

La chronologie d'apparition d'un œdème a une grande signification pronostique :

- L'œdème précoce apparu dès le $1^{\text {er }}$ jour, par exemple, est l'indice d'une irradiation à très haut niveau de dose. Le moment d'apparition de l'œdème entre le $1^{\text {er }}$ et le $21^{\text {e }}$ jour dépend de la dose sans qu'il soit, cependant, possible d'établir une corrélation entre la dose et la date d'apparition.

- L'œdème tardif (plusieurs semaines, mois ou années après l'exposition) précède et accompagne les poussées de vascularite. La durée de la crise œdémateuse est très variable. Elle dépend de l'importance des modifications physiopathologiques sous-jacentes; elle peut être de plusieurs semaines lorsqu'elle précède et accompagne une évolution prénécrotique. 


\section{Les phlyctènes (épidermite exsudative)}

Les phlyctènes constituent une lésion caractéristique de l'atteinte de la couche basale de la peau. Leur éclosion implique une dose minimale de l'ordre de 1200 à 2000 rads. Elles consistent en un soulèvement de la couche épidermique sous l'effet de l'exsudation d'une sérosité le plus souvent claire, qui confère à la phlyctène un caractère translucide (fig. 3). L'étude topographique et dimensionnelle des phlyctènes présente un intérêt dosimétrique; lorsqu'il existe un gradient de dose, la périphérie de la phlyctène correspond à une dose cutanée comprise entre 1200 et 2000 rads. II peut être intéressant lorsque la dosimétrie physique n'a fourni que des courbes isodoses relatives de caler l'isodose 1800 rads sur le pourtour de la phlyctène.

Dans les cas d'exposition à des rayonnements de haute énergie, on peut observer des phlyctènes au niveau de la surface de sortie.

La chronologie d'apparition des phlyctènes dépend de la dose reçue par la basale et présente donc un grand intérêt dosimétrique bien qu'il ne soit pas possible, dans l'état actuel de l'exploitation de nos données, d'établir une relation exacte entre la date d'apparition de la phlyctène et la dose. Un délai d'apparition d'une première phlyctène de 21 jours traduit un niveau de dose de 1200 à 2000 rads. Le délai d'apparition d'une phlyctène est d'autant plus court que la dose est plus élevée. Dans les cas d'exposition hétérogène des doigts d'une main, par un faisceau tangentiel de rayons $X$ par exemple, il est fréquent d'assister à l'éclosion successive des phlyctènes, les premières répondant aux doses les plus élevées. L'évolution des phlyctènes conduit à une ulcération superficielle. Cette ulcération peut :

- soit régresser et l'on peut observer une restauration cutanée avec ou sans séquelles dans un délai de quelques semaines à quelques mois, délai d'autant plus long que la phlyctène est apparue plus précocément ;

- soit évoluer vers la nécrose.

La phlyctène considérée isolément ne présente qu'un intérêt pronostique limité, car elle ne dépend que de la dose en surface et ne préjuge pas de la dose en profondeur. Par contre, si aux notions de délai d'apparition et d'évolution des phlyctènes on associe la notion d'énergie du rayonnement, l'intérêt pronostique devient très important.

\section{La nécrose}

L'évolution des radiolésions aiguës localisées s'effectue vers la nécrose lorsque le niveau de dose est supérieur à 2500 rads. Elle débute le plus souvent par une ulcération (fig. 4) plus ou moins profonde dont le fond est jaunâtre, recouvert d'un exsudat fibrineux; l'aspect peut se modifier, devenir noirâtre, avec assèchement. Ce dernier aspect s'observe, en particulier, au niveau des phalanges digitales.

L'installation de la nécrose est plus ou moins rapide; le délai d'apparition est de quelques semaines à quelques mois. Elle peut évoluer d'une seule tenue en quelques mois, ou en plusieurs temps échelonnés sur plusieurs années. Elle succède aux poussées de vascularite. Géné- 
ralement limitée à la masse de tissu irradié, elle peut, dans certains cas, s'étendre au-delà et prendre une allure extensive. Jamais réversible, elle appelle toujours une sanction chirurgicale.

\section{La sclérose}

La sclérose est une des réponses les plus fréquentes des tissus à Pagression radiologique. Elle se manifeste au niveau des tissus musculaires, tendineux et aponévrotiques et présente un caractère rétractile.

Elle peut apparaître assez tôt, dès les premières semaines, ou beaucoup plus tard, se stabiliser, et contribuer alors au processus de guérison et constituer une séquelle, ou à l'inverse présenter un caractère évolutif.

La sclérose rétractile précoce est le fait de doses élevées; elle est particulièrement spectaculaire au niveau des mains, notamment lors d'exposition à prédominance palmaire. Les doigts se recroquevillent et se fixent en flexion entraînant une ankylose rapide des articulations. Une telle situation pose de difficiles problèmes thérapeutiques et peut inciter à des actes chirurgicaux indépendamment de considérations radiologiques proprement dites.

La sclérose rétractile tardive se manifeste au bout de quelques mois ou de quelques années. Elle peut intéresser des volumes considérables, des structures profondes et constituer des lésions sans commune mesure avec l'aspect cutané, dans les cas où elle est induite par des rayonnements de haute énergie.

\subsubsection{Signes fonctionnels}

Parmi les nombreuses manifestations fonctionnelles de la maladie, deux présentent des caractères particuliers. Ce sont la sensation de chaleur initiale, et la douleur.

- La sensation de chaleur initiale, indépendante de la température de la source, a été rapportée 10 fois sur les 60 cas étudiés. La nature et l'énergie des rayonnements n'interviennent pas ; il s'agissait d'exposition :

$\begin{array}{ll}\text { - aux X } & 4 \text { fois } \\ \text { - au }{ }^{60} \text { Co } & 3 \text { fois } \\ \text { - aux électrons } & 2 \text { fois } \\ \text { - aux neutrons } & 1 \text { fois }\end{array}$

Par contre, le débit de dose joue un rôle fondamental. II est à noter qu'aucun cas d'exposition à des sources de ${ }^{192} \mathrm{Ir}$ n'a présenté le phénomène. Dans les cas d'exposition à des sources de ${ }^{60} \mathrm{Co}$, il s'agissait de préhension de sources de haute activité $(700 \mathrm{Ci}$ et $3700 \mathrm{Ci})$.

La douleur: Elle est constante. Les 60 sujets qui font l'objet de l'analyse ont présenté un syndrome douloureux. II s'agit toujours d'une sensation de brûlure dans le volume irradié. Elle est, dans la majorité des cas, très intense, permanente et présente des paroxysmes. Elle est particulièrement intolérable dans le cas de lésions profondes provoquées par 


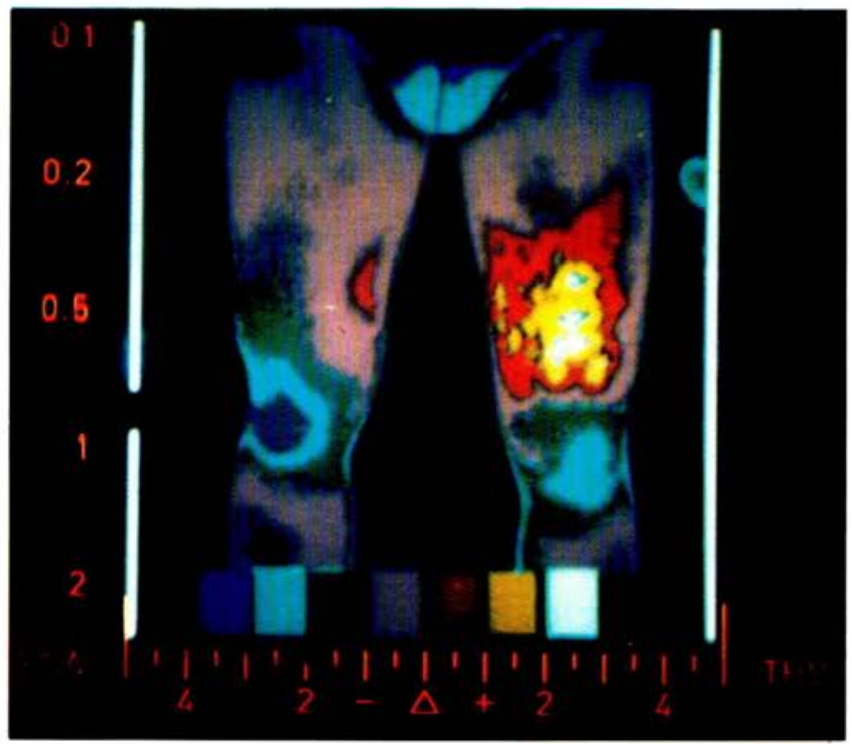

Fig. 1. - Thermographie $24 \mathrm{~h}$ apres irradiation accidentelle de la cuisse gauche par une source de ${ }^{192} \mathrm{Ir}$.

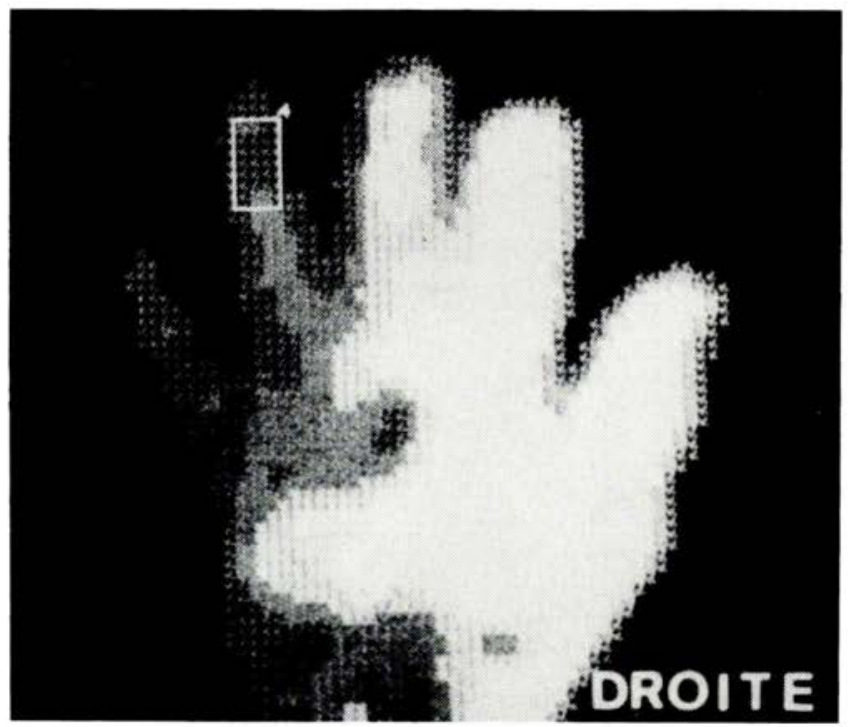

Fig. 2. Scintigramme vasculaire $11 \mathrm{j}$ après irradiation accidentelle de la main par une source de ${ }^{60} \mathrm{Co}$. 
Fig. 3. - Lésion phlycténulaire $4 \mathrm{j}$ après irradiation accidentelle par un faisceau de rayons $X$ émis sous une tension de $\mathbf{5 0} \mathbf{~ k V}$.
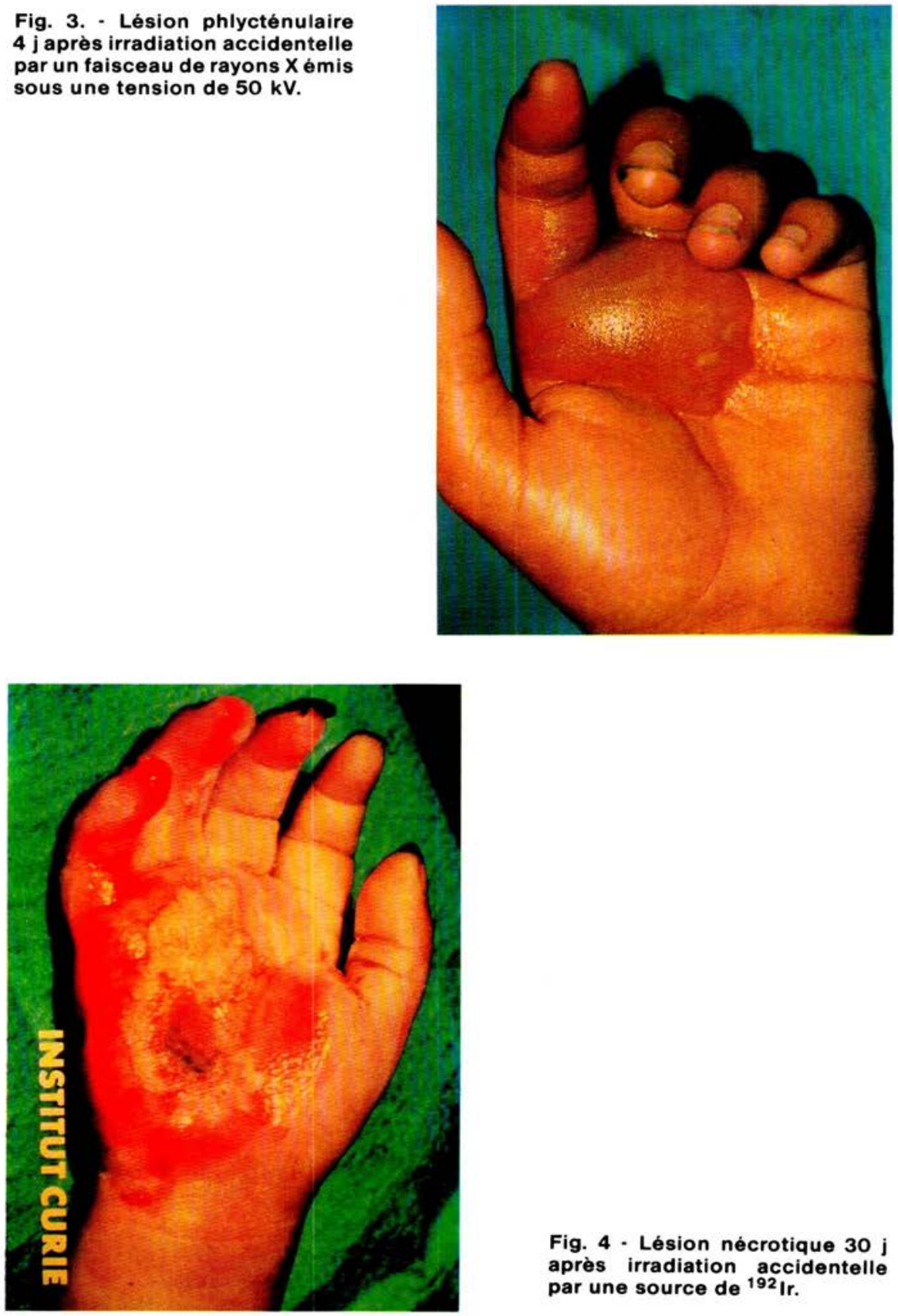

Fig. 4 - Lésion nécrotique 30 j après irradiation accidentelle par une source de ${ }^{192}$ Ir. 
des photons d'énergie élevée $\left({ }^{192} \mathrm{Ir}\right.$ et $\left.{ }^{60} \mathrm{Co}\right)$. Les sujets rapportent l'impression d'être "brûlés au chalumeau", la sensation d'écrasement ou de broyage des tissus, la sensation de décharges électriques au rythme fréquent. Lors de poussées de vascularite, la douleur permanente s'intensifie et des crises paroxystiques accompagnent les manifestations érythémateuses, œdémateuses, inflammatoires liées à de brusques réactions vasomotrices. La durée de la crise douloureuse intense est de plusieurs jours ; les poussées paroxystiques de l'ordre de $30 \mathrm{~min}$ à $1 \mathrm{~h}$ se répètent plusieurs fois par jour. Ces douleurs peuvent irradier en dehors du territoire lésé.

Le syndrome douloureux des radiolésions aiguës localisées résiste à la plupart des thérapeutiques médicales. Seule, l'excision des tissus lésés peut être efficace. Les indications chirurgicales étant difficiles à poser, et les décisions ne pouvant souvent être prises qu'après plusieurs semaines et parfois plusieurs mois d'évolution, la douleur peut constituer une préoccupation majeure. Elle conduit à l'administration prolongée d'opiacés avec les risques d'accoutumance.

Le syndrome douloureux, par son intensité, sa continuité, et sa durée, a un retentissement psycho-affectif qui peut induire des intentions suicidaires.

\subsection{Formes cliniques}

De nombreux paramètres conditionnent l'évolution clinique de la maladie et lui confèrent des caractères particuliers.

\subsubsection{Formes évolutives}

On peut distinguer en fonction de l'évolution :

- Une forme limitée à l'épidermite exsudative. Elle comporte des phases bien individualisées :

- érythème initial,

- phase de latence clinique,

- érythème secondaire et épithéliite exsudative,

- la restauration soit sans séquelles, soit avec séquelles.

- Une forme avec endothéliite vasculaire entre 6 et 18 mois. Le sujet peut présenter, le premier jour, un discret érythème fugace, mais après une phase de latence clinique totale de 20 jours, apparaît un érythème "tardif" immédiatement suivi d'une épithéliite exsudative. La guérison de cette épithéliite s'effectue en trois mois à partir à la fois des berges de l'ulcération et d'îlots centraux d'épithélialisation. Une guérison apparente se maintient jusqu'au $6^{*}$ mois, parfois davantage, jusqu'au $18^{e}$ mois. Alors, se déclare une ulcération nécrotique, torpide et extensive, qui implique une intervention chirurgicale.

- A côté de ces formes caractérisées par la chronologie d'apparition des signes d'épithéliite exsudative et d'endothéliite vasculaire, on observe pour des doses plus élevées, des formes contractées qui se caractérisent soit par un raccourcissement des phases et de leur intervalle, 
soit par une fusion des phases et une évolution d'une seule tenue qui conduit à une nécrose massive en quelques semaines.

\subsubsection{Formes liées aux caractères physiques de la source}

La nature et l'énergie des rayonnements jouent un rôle important. Dans un cas d'exposition d'un doigt à un faisceau étroitement collimaté d'électrons de $4 \mathrm{MeV}$, on a pu observer l'élimination d'un séquestre osseux 7 ans après l'exposition. Le sujet avait présenté initialement les signes observés habituellement après une exposition à niveau de dose élevé (érythème au $12^{\mathrm{e}}$ jour, phlyctène au $19^{\circ}$ jour, épithéliite exsudative et restauration apparemment ad integrum en 3 mois).

A titre de comparaison, dans un autre cas d'irradiation de la main par un faisceau d'électrons de très faible énergie, le sujet a accusé une sensation de chaleur lors de l'exposition ; II a présenté, dès le $3^{\bullet}$ jour, un érythème et une phlyctène. La guérison définitive était acquise au $4^{\mathbf{e}}$ mois. Dans ce cas, seules les couches superficielles de la peau étaient lésées.

La pénétration du rayonnement dans les tissus est liée à son énergie. Les lésions sont d'autant plus graves que l'énergie est plus élevée. Si l'on considère, par exemple, les cas d'irradiation par photons à dose élevée ayant entraîné des lésions sévères : dans les deux cas d'exposition des mains à des photons $X$ de 4 à $12 \mathrm{keV}$, la restauration a été obtenue après chirurgie conservatrice; dans les seize cas d'exposition des mains à des photons $X$ de 20 à $50 \mathrm{keV}$ ayant entraîné des lésions aiguës, la guérison a été obtenue avec ou sans séquelles, mais sans mutilation; par contre, dans tous les cas d'irradiation des mains par les photons du ${ }^{60} \mathrm{Co}$ $(1,17-1,33 \mathrm{MeV})$, qui sont au nombre de 8 , il a été nécessaire de pratiquer une amputation; dans les cas d'exposition des mains aux photons du ${ }^{192} \operatorname{Ir}(0,3 \mathrm{MeV})$, au nombre de 13 , il a été nécessaire de pratiquer une amputation 6 fois.

\subsubsection{Formes liées aux modalités chronologiques d'exposition}

- La plupart des radiolésions aiguës localisées ont pour origine des expositions de courte durée.

- Les expositions qui se caractérisent par un long étalement induisent des lésions torpides, chroniques, comparables à certaines séquelles de la radiothérapie. Lorsque le niveau de dose est très important, elles peuvent entraîner des lésions de nécrose ; c'est le cas pour un infirmier qui, pendant 20 ans, a utilisé un appareil de radioscopie et a participé à de nombreuses extractions de corps étrangers; il présentait, 10 ans après la cessation de ses activités, une vaste ulcération nécrotique chronique des trois premiers doigts de la main gauche qui a nécessité une amputation.

\subsubsection{Formes liées au volume et à la topographie}

Le volume des territoires irradiés conditionne l'évolution; une dose très élevée sur un petit volume d'un organe non vital n'a pas de mauvais pronostic, tandis qu'une dose de 1800 rads sur un volume important et intéressant des organes vitaux peut avoir un pronostic fatal. Une irradiation entraînant des lésions de la peau dans sa totalité est mortelle. 
La première de ces trois éventualités est illustrée par le cas d'un sujet qui a été exposé au niveau de la main droite à un faisceau de rayons $X$ étroitement collimaté, de très faible énergie (4 à $12 \mathrm{keV}$ ) mais de débit très élevé. II a présenté une ulcération de la paume de la main au niveau de la base des $2^{\circ}$ et $3^{\circ}$ métacarpiens, de petit volume $(30 \mathrm{~mm}$ sur $10 \mathrm{~mm}$, et quelques $\mathrm{mm}$ de profondeur), bien limitée, qui a pu être excisée dans sa totalité et guérie pratiquement sans séquelles après chirurgie réparatrice.

La dernière éventualité, atteinte du corps entier, est illustrée par le cas d'une malade qui a été exposée à une source de ${ }^{192} \mathrm{Ir}$ de $25 \mathrm{Ci}$ dans des conditions de géométrie et de chronologie complexes. Elle a présenté un syndrome d'irradiation globale aigü associé au syndrome cutané. L'épithéliite exsudative s'est manifestée d'abord sur la face antérieure du thorax, puis s'est étendue à la quasi-totalité du corps. En plus du syndrome cutané, la malade présentait des radiolésions des voies respiratoires et une aplasie médullaire. Le décès est survenu 26 jours après la fin de l'exposition.

Le siège de la lésion joue un rôle important; il conditionne l'évolution, le pronostic et la thérapeutique. Pour un même segment anatomique, les conséquences peuvent être très différentes selon la distribution topographique de la dose. Ainsi, au niveau de la cuisse, le pronostic est beaucoup plus sévère pour les brûlures de la face interne que pour celles de la face externe. De même, au niveau des mains, le pronostic est plus sévère pour les lésions distales que pour les lésions proximales. Ces faits sont illustrés par deux cas d'irradiation de cuisse et par deux cas d'irradiation de mains.

Deux sujets ont été exposés au niveau de la cuisse à une source de ${ }^{192} \mathrm{Ir}$. Dans le premier cas, le sujet a transporté pendant $4 \mathrm{~h}$ une source de ${ }^{192} \mathrm{Ir}$ de $15 \mathrm{Ci}$ dans la poche droite de sa veste ; il a présenté des lésions scléronécrotiques de la face externe de la cuisse qui ont épargné l'axe vasculaire ; après résection des tissus nécrosés et greffe, il a pu conserver son membre inférieur et récupérer une fonction très acceptable. A l'inverse, dans le deuxième cas, l'évolution a conduit à l'amputation. Le sujet a porté pendant $7 \mathrm{~h} 30$ une source de ${ }^{192} \mathrm{Ir}$ de $14 \mathrm{Ci}$ dans la poche antérieure gauche de sa combinaison. C'est la face antéro-interne de la cuisse qui a été exposée. Dès le premier jour, il a présenté un érythème et des paresthésies ; une épithéliite exsudative est apparue au $20^{\circ}$ jour, bientôt suivie d'une vaste ulcération nécrotique qui a évolué pendant plusieurs mois. Les lésions, de topographie interne, intéressaient l'artère fémorale au niveau de laquelle la dose était de 7400 rads; malgré une tentative de pontage artériel et de greffe de lambeau libre, il a été nécessaire de pratiquer une amputation.

L'importance de la topographie est encore plus sensible au niveau des mains. Les lésions distales sont beaucoup plus sévères que les lésions proximales. La plupart des sujets ayant subi une irradiation des doigts par les photons du ${ }^{192} \mathrm{Ir}$ ou du ${ }^{60} \mathrm{Co}$ ont dú subir une amputation.

A l'inverse, les lésions dues à une irradiation de la paume de la main peuvent, dans une large mesure, bénéficier de thérapeutiques chirurgicales conservatrices. C'est le cas d'un enfant de 3 ans et demi qui a présenté une profonde ulcération de la paume de la main gauche pour avoir manipulé une source de ${ }^{192}$ Ir de $25 \mathrm{Ci}$. 


\subsubsection{Formes associées à une irradiation globale aiguë}

Treize cas répondent à cette éventualité. Le pronostic vital est lié au syndrome d'irradiation globale. On peut distinguer :

- Les formes à irradiation globale prédominante. Trois sujets présentaient à la fois une aplasie médullaire et des brûlures. Deux d'entre eux n'ont pas survécu au syndrome d'irradiation globale. Pour l'un deux, la dose globale moyenne était de 1200 rads. Le troisième sujet pour lequel la dose globale moyenne était de 550 rads, présentait une surexposition du pied et de la jambe gauches ; la dose qui était de 5000 rads au niveau de l'avant-pied, était décroissante de bas en haut ; elle était de 1200 rads au niveau du pubis. Ce sujet a dû subir une amputation à mi-cuisse.

- Les formes à syndrome d'irradiation locale prédominant.

\subsubsection{Formes avec induction de cancer}

La complication classiquement la plus redoutée est l'induction de cancer. Elle peut survenir théoriquement chez les sujets qui ont guéri sans traitement chirurgical et chez les sujets ayant subi un traitement chirurgical conservateur sans excision de la totalité du tissu irradié. Le temps d'induction de ces cancers peut être très long, 10 à 20 ans. Pour l'ensemble des cas d'irradiation locale aiguë dont les premiers remontent à 1956, il n'a pas été observé de dégénérescence maligne.

Un seul cas de cancer induit a été constaté ; il s'agissait d'irradiation chronique chez un homme de 66 ans ayant pratiqué pendant 20 ans des radioscopies en exposant ses mains au faisceau de rayons $X$. L'épithélioma s'est déclaré 10 ans après la fin de la période d'exposition. Ce malade ne fait évidemment pas partie des 60 cas considérés.

\section{TRAITEMENT}

Le traitement des radiolésions aiguës localisées est complexe et difficile. D'abord, il vise à lutter contre l'infection, contre l'installation de troubles trophiques et d'ankylose, et contre la douleur. Mais lorsque se sont constituées des lésions scléronécrotiques, il faut avoir recours à la chirurgie qui consiste en chirurgie de propreté (résections limitées ou amputations larges), et en chirurgie conservatrice et de réparation (greffes et lambeaux pédiculés ou libres).

Le siège et le volume des tissus à réséquer sont dictés d'abord par les données dosimétriques, ensuite par des considérations techniques et fonctionnelles.

\section{CONCLUSIONS}

Les radiolésions accidentelles sont rares, mais elles peuvent être très sévères. Elles sont, le plus souvent, le fait d'une erreur humaine associée à un incident technique. 
La gravité de la maladie dépend de la dose délivrée aux tissus. Cette dose conditionne l'évolution et a une incidence sur la conduite thérapeutique. II est essentiel de la déterminer; le dosimétriste a donc besoin de connaître les circonstances physiques de l'exposition : caractéristiques physiques de rayonnement, conditions géométriques et durée. Les données fournies par les dosimètres d'ambiance ou individuels sont précieuses. Le médecin a besoin de savoir si le sujet irradié a présenté des manifestations précoces telles que sensation de chaleur, érythème ou œdème. Les données précoces des examens paracliniques étant essentielles, il est nécessaire que les sujets soient examinés en milieu spécialisé aussitôt que possible.

\section{RÉFÉRENCES}

Le lecteur trouvera dans "The Medical basis for radiation accident preparedness , proceedings of the REAC/TS International conference, Oak Ridge, October 18-20, 1979 (K.F. Hubner and Sh. A. Fry, Eds), Amsterdam : Elsevier, 1980", des informations récentes sur les problèmes posés par les irradiations accidentelles et de nombreuses références bibliographiques. 